\title{
THE SIZE EVOLUTION OF PASSIVE GALAXIES: OBSERVATIONS FROM THE WIDE-FIELD CAMERA 3 EARLY RELEASE SCIENCE PROGRAM*
}

\author{
R. E. Ryan, JR. ${ }^{1}$, P. J. McCarthy ${ }^{2}$, S. H. Cohen $^{3}$, H. YAN ${ }^{4}$, N. P. Hathi ${ }^{5}$, A. M. Koekemoer ${ }^{6}$, M. J. RutKowski ${ }^{3}$, \\ M. R. Mechtley ${ }^{3}$, R. A. Windhorst ${ }^{3}$, R. W. O’Connell ${ }^{7}$, B. Balick ${ }^{8}$, H. E. Bond ${ }^{6}$, H. Bushouse ${ }^{6}$, D. Calzetti ${ }^{9}$, \\ R. M. Crockett ${ }^{10}$, M. Disney ${ }^{11}$, M. A. Dopita ${ }^{12}$, J. A. Frogel ${ }^{13,14}$, D. N. B. Hall ${ }^{15}$, J. A. Holtzman ${ }^{16}$, S. Kaviraj ${ }^{10}$,

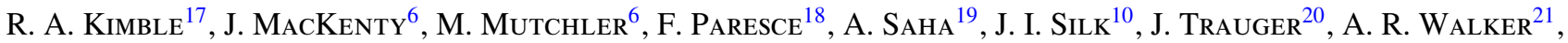 \\ B. C. Whitmore ${ }^{6}$, And E. Young ${ }^{22}$ \\ ${ }^{1}$ Physics Department, University of California, Davis, CA 95616, USA; rryan @ physics.ucdavis.edu \\ ${ }^{2}$ Observatories of the Carnegie Institute of Washington, Pasadena, CA 91101, USA \\ ${ }^{3}$ School of Earth and Space Exploration, Arizona State University, Tempe, AZ 85287, USA \\ ${ }^{4}$ Center for Cosmology and Astroparticle Physics, Ohio State University, Columbus, OH 43210, USA \\ ${ }^{5}$ Department of Physics and Astronomy, University of California, Riverside, CA 92521, USA \\ ${ }^{6}$ Space Telescope Science Institute, Baltimore, MD 21218, USA \\ ${ }^{7}$ Department of Astronomy, University of Virginia, Charlottesville, VA 22904, USA \\ ${ }^{8}$ Department of Astronomy, University of Washington, Seattle, WA 98195, USA \\ ${ }^{9}$ Department of Astronomy, University of Massachusetts, Amherst, MA 01003, USA \\ ${ }^{10}$ Department of Physics, University of Oxford, Oxford OX1 3PU, UK \\ ${ }^{11}$ School of Physics and Astronomy, Cardiff University, Cardiff CF24 3AA, UK \\ ${ }^{12}$ Research School of Astronomy and Astrophysics, The Australian National University, Weston Creek, ACT 2611, Australia \\ ${ }^{13}$ Galaxies Unlimited, Lutherville, MD 21093, USA \\ ${ }^{14}$ Astronomy Department, King Abdulaziz University, P.O. Box 80203, Jeddah 21589, Saudi Arabia \\ ${ }^{15}$ Institute for Astronomy, University of Hawaii, Honolulu, HI 96822, USA \\ ${ }^{16}$ Department of Astronomy, New Mexico State University, Las Cruces, NM 88003, USA \\ ${ }^{17}$ NASA, Goddard Space Flight Center, Greenbelt, MD 20771, USA \\ ${ }^{18}$ Istituto di Astrofisica Spaziale e Fisicia Cosmica, INAF, via Gobetti 101, 40129 Bologna, Italy \\ ${ }^{19}$ National Optical Astronomy Observatories, Tucson, AZ 85726, USA \\ ${ }^{20}$ NASA, Jet Propulsion Laboratory, Pasadena, CA 91109, USA \\ ${ }^{21}$ Cerro Tololo Inter-American Observatory, La Serena, Chile \\ 22 NASA, Ames Research Center, Moffett Field, CA 94035, USA \\ Received 2010 July 7; accepted 2012 January 18; published 2012 March 22
}

\begin{abstract}
We present the size evolution of passively evolving galaxies at $z \sim 2$ identified in Wide-Field Camera 3 imaging from the Early Release Science program. Our sample was constructed using an analog to the passive $B z K$ galaxy selection criterion, which isolates galaxies with little or no ongoing star formation at $z \gtrsim 1.5$. We identify 30 galaxies in $\sim 40 \operatorname{arcmin}^{2}$ to $H<25 \mathrm{mag}$. By fitting the 10-band Hubble Space Telescope photometry from $0.22 \mu \mathrm{m} \lesssim \lambda_{\text {obs }} \lesssim 1.6 \mu \mathrm{m}$ with stellar population synthesis models, we simultaneously determine photometric redshift, stellar mass, and a bevy of other population parameters. Based on the six galaxies with published spectroscopic redshifts, we estimate a typical redshift uncertainty of $\sim 0.033(1+z)$. We determine effective radii from Sérsic profile fits to the $H$-band image using an empirical point-spread function. By supplementing our data with published samples, we propose a mass-dependent size evolution model for passively evolving galaxies, where the most massive galaxies $\left(M_{*} \sim 10^{11} M_{\odot}\right)$ undergo the strongest evolution from $z \sim 2$ to the present. Parameterizing the size evolution as $(1+z)^{-\alpha}$, we find a tentative scaling of $\alpha \approx(-0.6 \pm 0.7)+(0.9 \pm 0.4) \log \left(M_{*} / 10^{9} M_{\odot}\right)$, where the relatively large uncertainties reflect the poor sampling in stellar mass due to the low numbers of highredshift systems. We discuss the implications of this result for the redshift evolution of the $M_{*}-R_{e}$ relation for red galaxies.
\end{abstract}

Key words: galaxies: evolution - galaxies: fundamental parameters - galaxies: structure

Online-only material: color figures

\section{INTRODUCTION}

Understanding the red, passively evolving galaxies at intermediate redshifts $(z \sim 2)$ is one of the outstanding challenges of galaxy evolution studies. Early expectations of a highluminosity phase associated with the formation of spheroids on a free-fall timescale (Eggen et al. 1962) have long given way to a framework in which the spheroids are assembled over an extended period of time. The identification of fairly high

\footnotetext{
* Based on observations made with the NASA/ESA Hubble Space Telescope, obtained from the Data Archive at the Space Telescope Science Institute, which is operated by the Association of Universities for Research in Astronomy, Inc., under NASA contract NAS 5-26555.
}

space densities of massive and passively evolving galaxies at $z \gtrsim 1.5$ (e.g., Glazebrook et al. 2004; Cimatti et al. 2004) revealed weaknesses in the early semi-analytic models of their formation. Large-scale surveys, both locally and out to $z \sim 1$, provide a fairly clear view of the evolution in the number density of red sequence galaxies and the global stellar mass density in passive systems (e.g., Bell et al. 2006; Faber et al. 2007; Brown et al. 2003, 2007).

Deep spectroscopic studies of red galaxies (e.g., McCarthy et al. 2004; Cimatti et al. 2006, 2008) estimated stellar ages consistent with early formation redshifts $\left(z_{\text {form }} \gtrsim 4\right)$, potentially at odds with the rapid evolution in stellar mass density at these epochs (e.g., Rudnick et al. 2003). It is now clear that the red 
sequence was in place at fairly early epochs (Bell et al. 2004; Bremer et al. 2006; Kriek et al. 2008), but evolved strongly in the $1 \lesssim z \lesssim 3$ era (e.g., Demarco et al. 2010) and likely since $z \sim 1$ as well (e.g., Faber et al. 2007). The emerging picture in which massive galaxies are assembled via mergers followed by a rapid quenching of star formation addresses many of the salient properties of the red sequence galaxies (Faber et al. 2007).

In light of these results it was quite surprising that high spatial resolution studies showed that the passive red galaxies at intermediate redshifts are systematically smaller than their likely present-day counterparts (e.g., Daddi et al. 2005; Trujillo et al. 2006; Toft et al. 2007). Observations of $1 \lesssim z \lesssim 2$ passive galaxies with either the Advanced Camera of Surveys (ACS) or the Near Infrared Camera and Multi-Object Spectrometer on the Hubble Space Telescope (HST) implied sizes that are a factor of $\sim 2$ smaller than equal mass red galaxies today (e.g., Longhetti et al. 2007; Cimatti et al. 2008; Damjanov et al. 2009), while observations of red galaxies at $z \gtrsim 2$ suggest even more dramatic evolution (e.g., van Dokkum et al. 2008; Saracco et al. 2010).

The primary interest in determining the characteristic sizes of passive galaxies is the insight that it provides into the dynamical state of the system. As one projection of the fundamental plane (Djorgovski \& Davis 1987; Dressler et al. 1987), the Kormendy relation (Kormendy 1977) provides a probe of the dynamics of hot stellar systems. The Kormendy diagram for the $z \gtrsim 1$ red sequence galaxies is nearly as tight as it is locally, but is offset in both surface brightness and size (e.g., Damjanov et al. 2009), even when the effects of passive fading of the stellar populations are taken into account. The compact sizes and high surface brightness of the red galaxies imply stellar densities in the centers of these galaxies that are two to three orders of magnitude higher than present-day massive red galaxies (e.g., van Dokkum et al. 2008; Damjanov et al. 2009).

The challenge in understanding these results stems from the apparent conflict between the requirement for a factor of $\sim 3$ growth in size during an epoch in which the stellar masses, population ages, and overall morphologies show little or no evolution. A number of models are under active discussion (e.g., Naab et al. 2006; Fan et al. 2008; Damjanov et al. 2009), but most involve large numbers of late-stage minor mergers that grow the galaxies in size without contributing large amounts of mass. While it is difficult to conclusively rule out various measurement biases which might give rise to this apparent trend (e.g., underestimated effective radii or overestimated masses), most interpretations are astrophysical in origin: (major or minor) merging occurring at different phases (e.g., Naab et al. 2006), or expansion due to a significant mass loss, either by an active galactic nucleus (Fan et al. 2008) or stellar winds (Damjanov et al. 2009). Recently, Hopkins et al. (2010) presented a semi-analytic model based on high-resolution hydrodynamic simulations (Cox et al. 2006), which incorporates several astrophysical and observational mechanisms for this trend, and concluded that for galaxies with a stellar mass of $M_{*} \geqslant 10^{11} M_{\odot}$, the late-stage minor merging is the dominant mechanism, accounting for $\sim 50 \%$ of the apparent size evolution. Certainly many of these high-redshift systems do in fact show merger-like features (e.g., van Dokkum \& Brammer 2010) or have (multiple) companions (e.g., Bell et al. 2006), and so merging is an attractive explanation for the observed size evolution.

The merging scenario implies that some non-negligible fraction ( $\lesssim 10 \%$; Hopkins et al. 2009) of compact galaxies should remain at $z \sim 0$. However, Trujillo et al. (2009) find that only
$0.03 \%(=48 / 152,083)$ of galaxies at $z \sim 0.2$ have stellar densities comparable to these high-redshift galaxies, arguing against the merger scenario. To further confound the issue, these galaxies are generally young ( $\sim 3 \mathrm{Gyr}$ old $)$, giving an approximate formation redshift of $z_{\text {form }} \sim 0.3$, suggesting that they are not the relics of the early universe, but formed from gas-rich, recently merged disks. Conversely, Saracco et al. (2010) find that $\sim 62 \%$ $(=21 / 34)$ of their galaxies at $1<z_{\mathrm{spec}}<2$ are within $1 \sigma$ of the local $R_{e}-M_{*}$ relation (Shen et al. 2003). Based on this finding, they suggest that the compact high-redshift galaxies are not the progenitors of large field galaxies, but of compact brightest cluster galaxies. The absence of compact early-type galaxies at $z \sim 0$ (e.g., Taylor et al. 2010) seems to suggest that the simple picture of galaxy merging with some mass-loss expansion is not completely correct, in stark contrast to the simulated results (e.g., Naab et al. 2009; Hopkins et al. 2010). In this paper, we present results from the Early Release Science (ERS) program with Wide-Field Camera 3 (WFC3) which suggest that the size evolution from $z \sim 2$ to the present depends on stellar mass. This finding should provide an additional observational constraint to further refine the theoretical models.

This paper is organized as follows. In Section 2, we describe the observations and ancillary data, in Section 3 we define our sample, in Section 4 we detail our stellar population modeling and morphological analysis, in Section 5 we describe our massdependent size evolution model, in Section 6 we discuss several key results, and in Section 7 we give a brief summary of this work with thoughts for future surveys. Throughout this paper, we assume a $\Lambda$ CDM cosmology with $\Omega_{0}=0.27, \Omega_{\Lambda}=0.73$, and $H_{0}=73 \mathrm{~km} \mathrm{~s}^{-1} \mathrm{Mpc}^{-1}$ (Spergel et al. 2003) and will quote all magnitudes in the $\mathrm{AB}_{v}$ magnitude system (Oke \& Gunn 1983).

\section{OBSERVATIONS}

\subsection{The Wide-Field Camera 3 Early Release Science}

For this work, we analyze the ERS (PropID: 11359, PI: R. W. O'Connell) observations conducted with the WFC3 recently installed on the HST. This field covers roughly the northern $40 \operatorname{arcmin}^{2}$ of the Great Observatories Origins Deep Survey southern field (GOODS-S; Giavalisco et al. 2004), and leverages the existing ACS optical data with near-ultraviolet (NUV) and near-infrared (NIR) data with equivalent space-based imaging. The main details regarding the data collection, reduction, calibration, and mosaicking are presented by Windhorst et al. (2011). We briefly summarize the observational aspects critical to this work.

The ERS program utilizes the two complementary modes of the WFC3: UVIS and IR channels. The data were collected between 2009 September and November and form a $2 \times 4$ and $2 \times 5$ mosaic, respectively. The final data set consists of $\sim 40 \mathrm{arcmin}^{2}$ of 10-band HST imaging covering $2250 \AA$ to $1.6 \mu \mathrm{m}$ in wavelength. The v2.0 GOODS high-level science products had an original pixel scale of 30 mas and were $3 \times 3$ block summed to produce science and weight maps of equal pixel scale to the WFC3 observations (see Windhorst et al. 2011, for a more detailed discussion on the rebinning process and its motivation). Therefore, our final HST mosaics have 90 mas pixels. We refer hereafter to the 10-band WFC3 and ACS filter set in the F225W, F275W, F336W, F435W, F606W, F775W, F850LP, F098M, F125W, and F160W as $U_{1} U_{2} U_{3} B V i^{\prime} z^{\prime} Y_{s} J H$ to simplify the bandpass notation. 


\subsection{Source Catalogs}

We created source catalogs using SExtractor (Bertin \& Arnouts 1996) with the $H$-band mosaic as the detection image and each of the HST mosaics as measurement images. We use the weight maps produced by MultiDrizzle (Koekemoer et al. 2002), modified to account for correlated pixel noise (Dickinson et al. 2004). For object detection, we require a minimum of 5 connected pixels each greater than $0.6 \sigma$ above the local background and apply a 3 pixel Gaussian smoothing filter. For deblending, we adopt a contrast parameter of $10^{-4}$ with 64 sub-thresholds. For photometry, we adopt the MAG_AUTO measurements with a Kron factor of 2.5, and minimum object radius of 3.5 pixels, which reliably recover total fluxes to within $\sim 6 \%$ (Bertin \& Arnouts 1996). We use the AB zero points given by Kalirai et al. (2009a, 2009b) for the UVIS and IR data, respectively.

\section{SAMPLE SELECTION}

We select our passively evolving galaxies using a variation on the standard $\mathrm{p} B z K$ selection (Daddi et al. 2004), designed to identify galaxies at $z \sim 2$ with little ongoing star formation and stellar population ages of $\sim 1 \mathrm{Gyr}$ (Daddi et al. 2005). However, to take full advantage of our high spatial resolution and significantly deeper ERS data set, and avoid issues associated with cataloging images with markedly different angular resolution, we use our $H$-band imaging instead of the ground-based $K$-band images. Therefore, we modify the classical $\mathrm{p} B z K$ selection criteria to an equivalent $\mathrm{p} B z H$ scheme:

$$
\begin{gathered}
\left(z^{\prime}-H\right)-\left(B-z^{\prime}\right)<-0.2-\langle(H-K)\rangle \mathrm{mag}, \\
\left(z^{\prime}-H\right)>2.5-\langle(H-K)\rangle \mathrm{mag} .
\end{gathered}
$$

As these $\mathrm{p} B z K$ galaxies have maximally old, passively evolving systems, we derive a typical color of $\langle(H-K)\rangle=0.7 \mathrm{mag}$ based on a stellar population with an instantaneous star formation history formed at $z_{\text {form }}=10$ based on S. Charlot \& G. Bruzual (2007, in preparation) and Bruzual (2007, hereafter CB07). In Figure 1, we show the $\left(B-z^{\prime}\right)$ and $\left(z^{\prime}-H\right)$ colors with these criteria illustrated as a shaded polygon. To rule out any potential image artifacts associated with the ERS field edges, we require the objects to be in the portion of the $H$-band mosaic which received the full complement of two orbits per pointing. We restrict the $H$-band magnitude to a relatively conservative limit of $H \leqslant 25 \mathrm{mag}$ to ensure a complete and reliable sample (Windhorst et al. 2011). With these requirements, we identify a final sample of 30 galaxies in $43.1 \mathrm{arcmin}^{2}$ and present their photometry (Table 1). Of these galaxies, 15 objects have very little (or no) flux in the $B$ band. To highlight their red colors and visual morphologies, we show a 4.'5, three-color stamp of each object in Figure 2.

We show the source counts of $B z H$ galaxies in Figure 3 . The counts plateau around $H \simeq 22.5 \mathrm{mag}$, which is roughly 4 mag brighter than the $H$-band completeness limit (Windhorst et al. 2011). Furthermore, since the GOODS $z^{\prime}$-band imaging is complete to $z^{\prime} \sim 27.3 \mathrm{mag}$ (Dahlen et al. 2010) and our effective color limit is $\left(z^{\prime}-H\right) \geqslant 1.8 \mathrm{mag}$, this plateau is unlikely due to incompleteness in the $z^{\prime}$-band data, suggesting that the faint end of their luminosity function is relatively flat or declining at low luminosities. Stutz et al. (2008) note a similar dearth of low-mass systems $\left(\lesssim 2 \times 10^{10} M_{\odot}\right)$, further supporting a flat or declining faint-end slope. At the bright end $(H \lesssim 22 \mathrm{mag})$, our

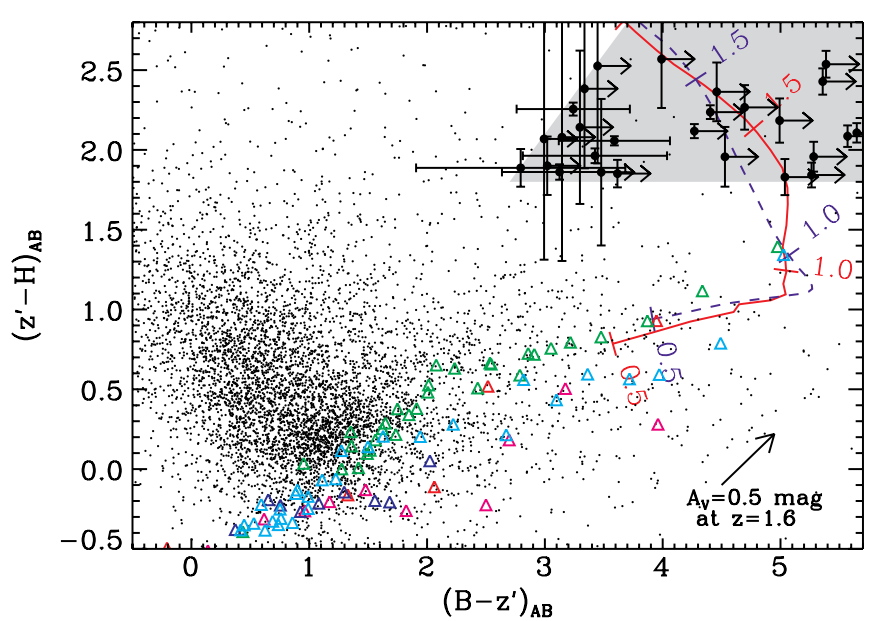

Figure 1. $B z H$ object selection. The small dots represent all objects detected and measured by SExtractor. The gray area is the color selection region defined by Equations (1) and (2) with a typical color of $\langle(H-K)\rangle=0.7 \mathrm{mag}$. We identify 30 galaxies strictly meeting the $B z H$ color criteria, for objects which were undetected in the $B$ band, we show the $5 \sigma$ point-source upper limits on the $\left(B-z^{\prime}\right)$ color (there are three objects off the plot). The few objects in the shaded selection region which are not in our sample either fall below our flux limit of $H<25 \mathrm{mag}$ or did not receive the full exposure time. We show a maximally old population with an instantaneous burst of star formation and the canonical (Coleman et al. 1980) elliptical galaxy template as solid red and dashed blue lines, respectively. We indicate redshift tick marks and values in the corresponding color. The arrow in the lower right corner shows the expected reddening for $A_{V}=0.5 \mathrm{mag}$ in the rest frame at $z=1.6$ for the Calzetti et al. (1994) dust law. We show the positions of Galactic stars from the Pickles (1998) library as open triangles and the color represents the luminosity class (I: magenta, II: red, III: green, IV: blue, V: cyan).

(A color version of this figure is available in the online journal.)

survey and modified $\mathrm{p} B z K$ selection method produces source counts consistent with Lane et al. (2007), based on the UKIRT Infrared Deep Sky Survey (UKIDSS) Ultra Deep Survey (UDS). While the UKIDSS/UDS survey is much wider $\left(\sim 0.6 \mathrm{deg}^{2}\right)$, the ERS data push $\gtrsim 2 \mathrm{AB}$ mag deeper into a regime not routinely possible from the ground.

\section{ANALYSIS}

\subsection{Surface Brightness Models}

To determine the rest-frame optical morphologies of our galaxies, we model the two-dimensional light distribution in the $H$ band using GalFit (Peng et al. 2002). We determine an empirical point-spread function (PSF) from a median stack of 75 stars identified in the field, based on their full width at halfmaximum (FWHM) and brightness determined by SExtractor (Windhorst et al. 2011). We fit the standard Sérsic profile:

$$
\Sigma(r)=\Sigma_{e} e^{-b_{n}\left[\left(r / r_{e}\right)^{1 / n}-1\right]},
$$

where $r_{e}$ is the effective radius, ${ }^{23} \Sigma_{e}$ is the surface brightness at the effective radius, $n$ is the Sersic index, and $b_{n}$ is a constant found by numerically solving $\Gamma(2 n)=2 \gamma\left(2 n, b_{n}\right)$ with $\Gamma(x)$ and $\gamma(x, y)$ representing the gamma and incomplete gamma functions, respectively. Since GalFit is minimizing a goodness-of-fit statistic, modeling the uncertainty in each pixel is critical for meaningful estimate of the uncertainty in each of the fit parameters. Therefore, we transform the scaled weight maps produced by MultiDrizzle into uncertainty maps and

\footnotetext{
${ }^{23}$ We refer to the effective radius as $r_{e}$ when in angular units and as $R_{e}$ when converted to physical units.
} 
Table 1

$B z H$ Sample and HST Photometry

\begin{tabular}{|c|c|c|c|c|c|c|c|c|c|c|}
\hline ID & $\begin{array}{l}\text { R.A. }^{a} \\
(\mathrm{~h} \mathrm{~m} \mathrm{~s})\end{array}$ & $\begin{array}{l}\text { Decl. }^{\mathrm{a}} \\
\left({ }^{\circ}{ }^{\prime \prime \prime}\right)\end{array}$ & $\begin{array}{c}B \\
(\mathrm{mag})\end{array}$ & $\begin{array}{c}V \\
(\mathrm{mag})\end{array}$ & $\begin{array}{c}i^{\prime} \\
(\mathrm{mag})\end{array}$ & $\begin{array}{c}z^{\prime} \\
(\mathrm{mag})\end{array}$ & $\begin{array}{c}Y_{s} \\
(\mathrm{mag})\end{array}$ & $\begin{array}{c}J \\
(\mathrm{mag})\end{array}$ & $\begin{array}{c}H \\
(\mathrm{mag})\end{array}$ & Notes \\
\hline 08 & 33228.02 & 274031.2 & $7.52 \pm 0.49$ & $26.18 \pm 0.12$ & $.18 \pm 0.08$ & $24.40 \pm 0.05$ & $23.77 \pm 0.03$ & $22.87 \pm$ & 1 & \\
\hline 06 & 33223.27 & -274045.8 & $>28.11$ & $27.60 \pm 0.53$ & $6.12 \pm 0.23$ & $25.36 \pm 0.14$ & $25.16 \pm 0.17$ & $3.66 \pm 0.03$ & $23.10 \pm 0.02$ & $\cdots$ \\
\hline 1696 & 33242.08 & -274141.2 & $>27.36$ & $>27.29$ & $26.69 \pm 1.12$ & $26.16 \pm 0.77$ & $25.47 \pm 0.32$ & $24.98 \pm 0.14$ & $24.08 \pm 0.08$ & $\cdots$ \\
\hline 2227 & 33242.34 & -274204.0 & $>27.55$ & $5.85 \pm 0.63$ & $4.53 \pm 0.13$ & $24.50 \pm 0.14$ & $23.86 \pm 0.07$ & $22.91 \pm 0.02$ & 2.32 & $\ldots$ \\
\hline 2377 & 33225.03 & -274209.6 & $>28.10$ & $27.80 \pm 0.67$ & $27.68 \pm 1.04$ & $27.43 \pm 1.00$ & $26.44 \pm 0.42$ & $25.42 \pm 0.11$ & $24.52 \pm 0.06$ & X-ray ID $226^{b}$ \\
\hline 2749 & 33214.92 & -274221.9 & $>27.23$ & $26.40 \pm 0.44$ & $24.92 \pm 0.19$ & $24.64 \pm 0.19$ & $24.67 \pm 0.19$ & $23.22 \pm 0.03$ & $22.69 \pm 0.03$ & $\cdots$ \\
\hline 2750 & 33214.88 & -274223.3 & $>27.26$ & $25.70 \pm 0.22$ & $24.56 \pm 0.14$ & $23.92 \pm 0.09$ & $23.95 \pm 0.09$ & $22.46 \pm 0.02$ & $21.97 \pm 0.01$ & X-ray ID $145^{b}$ \\
\hline 2871 & 33231.09 & -274226.6 & $>28.23$ & $26.85 \pm 0.22$ & $25.78 \pm 0.14$ & $24.91 \pm 0.08$ & $24.17 \pm 0.05$ & $3.39 \pm 0.02$ & $23.07 \pm 0.02$ & $\ldots$ \\
\hline 3000 & 33243.93 & -274232.4 & $6.23 \pm 0.4$ & $.12 \pm 0.13$ & $3.61 \pm 0.06$ & $22.64 \pm 0.03$ & $1.99 \pm 0.02$ & $1.07 \pm$ & 20.59 & $\cdots$ \\
\hline 3152 & 33226.05 & -274236.6 & $>27.96$ & $27.21 \pm 0.44$ & $24.93 \pm 0.09$ & $23.93 \pm 0.05$ & $23.31 \pm 0.02$ & $22.15 \pm$ & 21.66 & $\ldots$ \\
\hline 3237 & 33235.91 & -274240.9 & & & & & & & & $\ldots$ \\
\hline 3360 & 33230.58 & -274243.4 & $>28.06$ & & & & & & & $\cdots$ \\
\hline 3376 & 33235.13 & -274237.0 & $>28.04$ & $27.95 \pm 0.74$ & $26.80 \pm 0.45$ & $26.69 \pm 0.48$ & & & & $\ldots$ \\
\hline 3471 & 33227.94 & -274245.7 & $28.29 \pm 1.34$ & $26.29 \pm 0.17$ & $4.75 \pm 0.07$ & $24.02 \pm$ & $23.48 \pm$ & $22.35 \pm$ & 21.91 & $\ldots$ \\
\hline 3488 & 33225.65 & -274246.8 & $>27.97$ & $>28.20$ & $>27.63$ & $26.95 \pm 0.70$ & $26.01 \pm$ & $25.38 \pm$ & 24.93 & $\cdots$ \\
\hline 3551 & 33236.28 & -274249.4 & $27.78 \pm 1.42$ & $.05 \pm 0.24$ & $+.32 \pm 0.0$ & $23.38 \pm 0.04$ & $22.78 \pm$ & $21.63 \pm$ & 21.14 & $\ldots$ \\
\hline 3812 & 33236.66 & -274258.5 & $>28.02$ & $26.05 \pm$ & $.87 \pm$ & $5 \pm 0.04$ & $23.35 \pm 0$ & $22.23=$ & 21.74 & $\ldots$ \\
\hline 148 & 33244.97 & -274309.1 & $27.33 \pm 0.48$ & & & & & & & $\cdots$ \\
\hline 173 & 33241.24 & -274309.7 & & & & & $23.91 \pm 0$ & & 2 & $\cdots$ \\
\hline 4324 & 33231.32 & -274316.1 & $>27.48$ & $5.23 \pm 0$ & $24.65 \pm$ & $23.85 \pm 0$ & $.23 \pm 0$ & $22.25 \pm$ & $21.77=$ & $\cdots$ \\
\hline 4327 & 33159.71 & -274315.5 & $>28.40$ & $28.48 \pm 1.29$ & $26.81 \pm 0$ & $26.89 \pm 0.61$ & $25.90 \pm$ & $25.47 \pm$ & 24.37 & $\cdots$ \\
\hline 4534 & 33229.99 & -274322.7 & $>28.07$ & $27.44 \pm 0.51$ & $25.42 \pm 0.13$ & $24.37 \pm 0.06$ & $23.84 \pm 0.04$ & $22.74 \pm 0.01$ & $22.26 \pm 0.01$ & $\ldots$ \\
\hline 4648 & 33226.10 & -274326.7 & $28.39 \pm 1.28$ & $26.60 \pm 0.23$ & $25.47 \pm 0.13$ & $24.77 \pm 0.08$ & $24.29 \pm 0.07$ & $23.49 \pm 0.02$ & $22.92 \pm$ & $\ldots$ \\
\hline 4846 & 33225.93 & -274331.1 & $>28.28$ & $>28.37$ & $26.45 \pm 0.31$ & $26.23 \pm 0.31$ & $25.28 \pm 0.17$ & $24.08 \pm 0.04$ & $23.67 \pm 0.03$ & $\cdots$ \\
\hline 4921 & 33202.44 & -274335.8 & $>28.03$ & $>28.14$ & $>27.58$ & $26.98 \pm 0.75$ & $27.88 \pm 1.69$ & $26.13 \pm 0.23$ & $24.91 \pm 0.10$ & $\cdots$ \\
\hline 5410 & 33232.11 & -274355.3 & $>27.22$ & $5.33 \pm 0.38$ & $.26 \pm 0.2$ & & & $23.87 \pm 0.06$ & & $\cdots$ \\
\hline 5529 & 33201.77 & -274401.1 & $27.56 \pm 0.88$ & $28.02 \pm 1.16$ & $25.76 \pm 0.24$ & $24.76 \pm 0.12$ & $24.39 \pm 0.09$ & $23.46 \pm 0.03$ & $22.87 \pm$ & $\cdots$ \\
\hline 5685 & 33212.78 & -274407.7 & $27.98 \pm 1.78$ & $27.70 \pm 1.10$ & $25.17 \pm 0.18$ & $24.95 \pm 0.18$ & $24.17 \pm 0.09$ & $23.44 \pm 0.03$ & $23.06 \pm 0.03$ & $\ldots$ \\
\hline 5735 & 33202.83 & -274409.7 & $>28.05$ & $26.80 \pm 0.29$ & $26.88 \pm 0.52$ & $26.52 \pm 0.45$ & $26.10 \pm 0.33$ & $25.80 \pm 0.17$ & $24.66 \pm 0.08$ & $\cdots$ \\
\hline 6845 & 33206.57 & -274514.0 & $>27.57$ & $27.81 \pm 1.15$ & $25.93 \pm 0.34$ & $25.06 \pm 0.18$ & $24.80 \pm 0.15$ & $23.44 \pm 0.03$ & $22.69 \pm 0.02$ & \\
\hline 7202 & 33206.40 & -274554.7 & $7.10 \pm 0.61$ & $25.76 \pm 0.15$ & $24.78 \pm 0.10$ & $23.67 \pm 0.05$ & $23.16 \pm 0.03$ & $22.37 \pm 0.01$ & $21.71 \pm 0.01$ & X-ray ID $82^{b}$ \\
\hline
\end{tabular}

Notes.

a Coordinates refer to the $\mathrm{J} 2000$ epoch.

b X-ray identifications are from Luo et al. (2008).

explicitly include the shot noise from the objects. We perform all fits with GalFit in units of counts using the $H$-band mosaic effective exposure time of $5017.7 \mathrm{~s}$.

We excise an $81 \times 81$ pixel $^{2}$ stamp centered on each galaxy; this size was chosen as a compromise between a sufficient number of sky pixels for a robust sky estimation by GalFit, and the number of (generally unassociated) nearby galaxies. Neighboring galaxies can bias the parameter estimation of the primary galaxy, therefore one must carefully mask out the unmodeled objects, or simultaneously fit all the objects in the stamp. We opt for the latter, since it avoids the ambiguities in pixel masking, and permits the flux in a given pixel to be represented as the sum of independent components. However, without good initial conditions, the GalFit algorithm may not converge to a meaningful solution, since the number of degrees of freedom can be very large. Therefore, to estimate better initial conditions for each galaxy in the stamp and ultimately ensure convergence in the final simultaneous solution, we fitted the two-dimensional light profiles of the primary $B z H$ galaxy in question and any neighboring galaxies in a multi-stage process as follows.

1. We identify all neighboring galaxies and their associated pixels with SExtractor, using the same settings mentioned in Section 2.2. Any sources with FWHM_IMAGE ${ }_{H} \leqslant 1$ are eliminated from the SExtractor segmentation maps and object catalogs, since they are likely cosmic rays or other artifacts. These objects are masked in all subsequent fitting processes.

2. The pixels of any galaxy whose isophotes are truncated (based on the SExtractor flags) are masked, by setting the uncertainty maps to $10^{10}$ ADU. These galaxies are no longer considered in the GalFit process.

3. We model the light distribution of each remaining galaxy (including the primary $B z H$ galaxy) individually, while masking all the pixels associated with every other galaxy. For any neighboring galaxy with semi-minor axis of B_IMAGE $_{H} \leqslant 1$ pixel from SExtractor, we switch from fitting a Sérsic profile to a PSF model, in order to eliminate degenerate degrees of freedom. Our results are robust to the choice of semi-minor axis limit, provided that we do not permit it to be larger than the size of the empirical PSF (discussed in more detail below). It is important to note that this step is only present to get reasonably accurate initial conditions for subsequent simultaneous object fitting.

4. We refit a GalFit model, which contains a combination of point sources and Sérsic profiles, to the stamp as a whole using the results from the previous step as the initial guesses.

In a few cases, we manually masked diffraction spikes or stellar halos clearly associated with foreground stars, which were just 

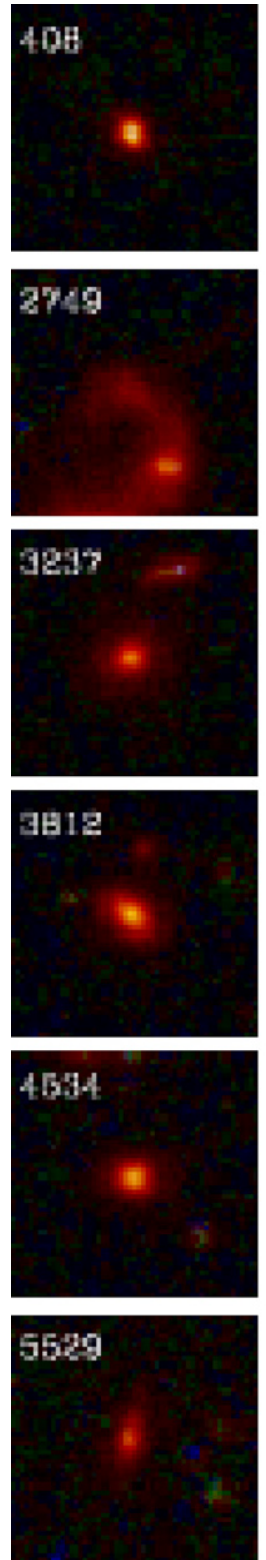
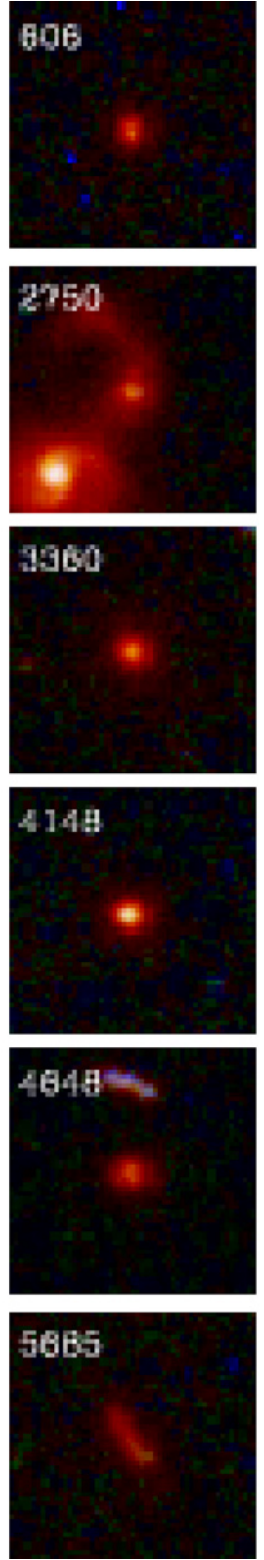
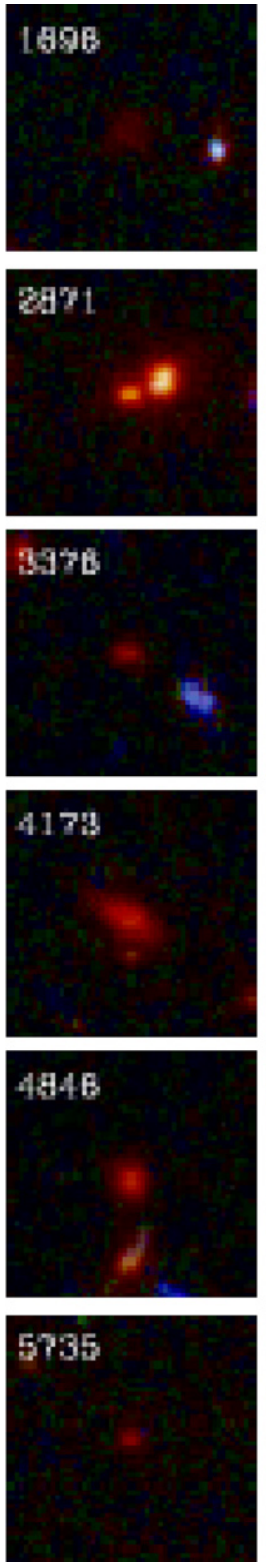
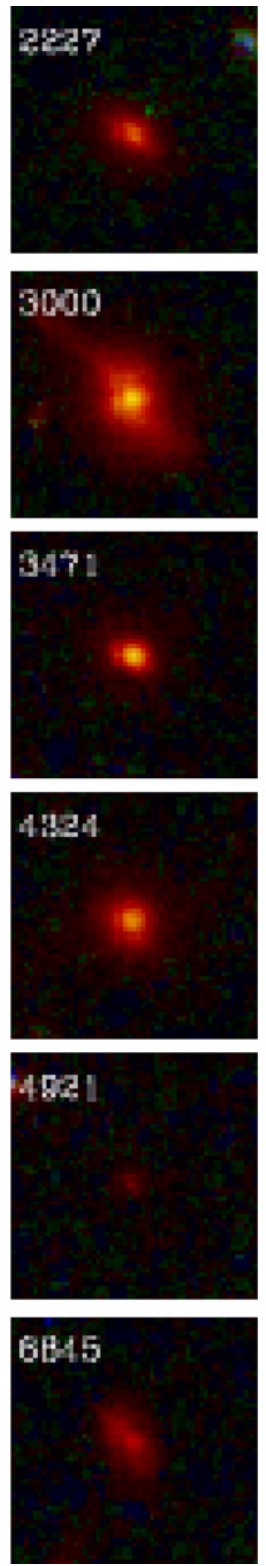
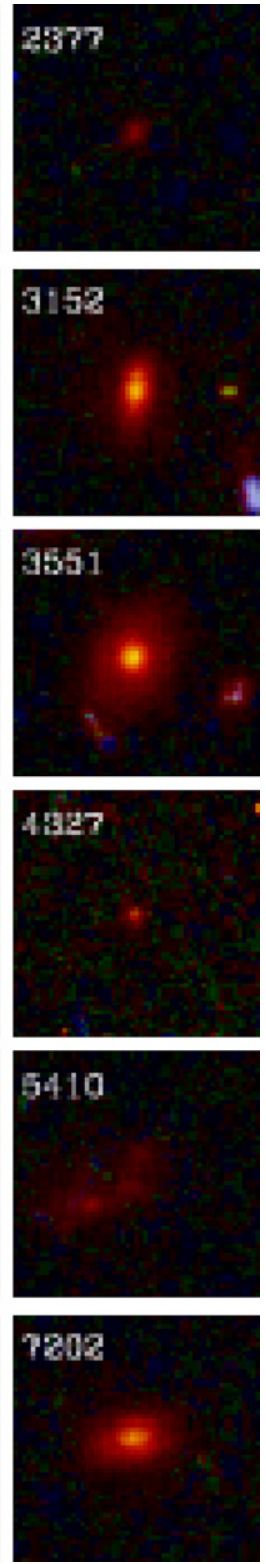

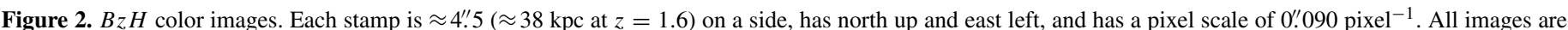

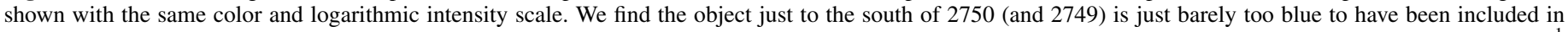

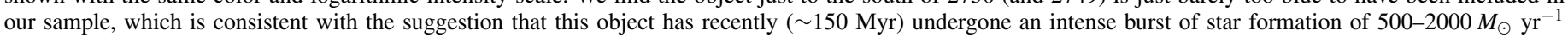
(van Dokkum \& Brammer 2010; Ferreras et al. 2011).

outside the field of view of the stamp before proceeding through the above procedure.

For the individual and simultaneous fits, we placed constraints on various GalFit parameters to prevent the algorithm from diverging into an unphysical regime. We constrained the centroid of any component to be within the $\pm 2 \sigma$ of the centroid determined by SExtractor, and the total magnitude to be $35 \leqslant H_{\text {tot }} \leqslant$ MAG_AUTO $H_{H}-2$ mag. In general, these constraints are so weak that they generally play no role in the fitting whatsoever, but they reduce the sensitivity to the pixel masking with the segmentation maps. We additionally constrain the Sérsic index and effective radius to be $0.01 \leqslant n \leqslant 8$ and $0.01 \leqslant r_{e} / A_{-}$IMAGE $\leqslant 5$, respectively. These constraints are considerably stronger, and we recognize that the model is likely incorrect when GalFit converges to a solution which is on these boundaries. These cases are rare, and generally a sign that additional astrophysical components are needed (e.g., bulge/disk separately, nuclear point sources, or merger signatures), that the frame was inappropriately sized, that the object was unresolved, and/or that there was some additional light component or image defect present in the image (e.g., diffraction spikes, stellar halos, and/or cosmic rays).

As our primary interest here is on the sizes of these galaxies, it is imperative that we ensure the effective radii are robustly measured. While GalFit reliably determines the random uncertainty which follows from the maximum likelihood analysis, the total uncertainty should include a systematic term as well. To estimate the contribution from the systematic uncertainty, we construct a grid of simulated galaxies with brightnesses $20 \mathrm{mag} \leqslant H \leqslant 25 \mathrm{mag}$, effective radii 0.5 pixel $\leqslant r_{e} \leqslant 5.5$ pixel, and a fixed Sérsic index of $n=4$ (for simulation only). These galaxies are convolved with the PSF and embedded in a blank region of the $H$-band mosaic. We then fitted these simulated galaxies with GalFit using the above procedure and find that 


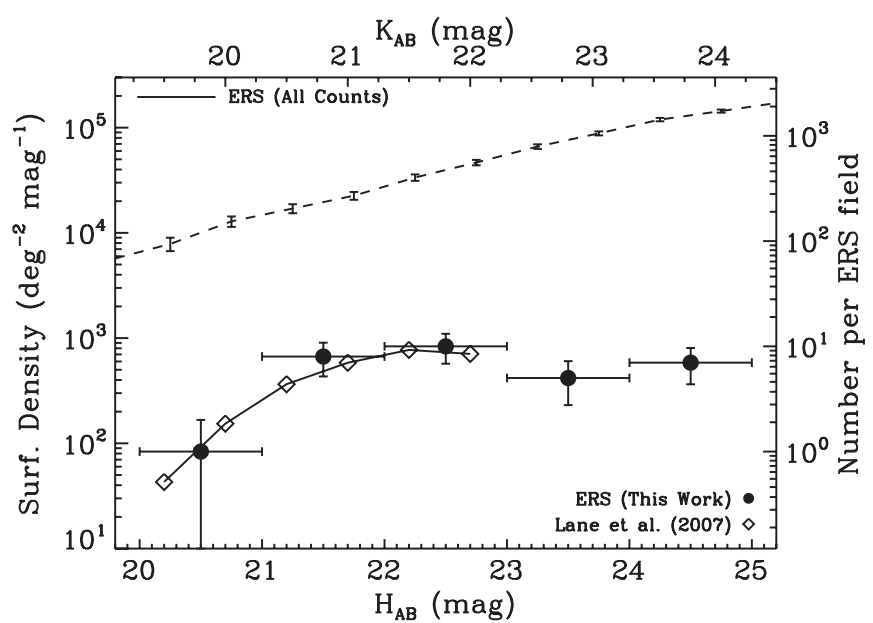

Figure 3. $B z H$ galaxy counts. We show the differential surface density of galaxies selected based on the usual $B z K$ color criteria (Daddi et al. 2004) and a typical color of $\langle(H-K)\rangle=0.7 \mathrm{mag}$, assuming an instantaneous burst of star formation and passive evolution from $z_{\text {form }}=10$. For comparison, we show the $B z K$ object counts from Lane et al. (2007) from the UKIDSS/UDS survey as open symbols, which were derived from a strict $B z K$ selection. The upper axis is simply a transformation using the assumed $\langle(H-K)\rangle$ color and the right axis applies to our objects. To demonstrate that the apparent plateau in the $B z H$ counts $H_{\mathrm{AB}} \sim 22 \mathrm{mag}$ is not due to sample incompleteness in the $H$ band, we show the total source counts from the ERS data as a dashed line (Windhorst et al. 2011). Moreover, this plateau is unlikely related to the $z^{\prime}$-band data, since two of our selection criteria $\left(H \leqslant 25 \mathrm{mag}\right.$ and $\left.\left(z^{\prime}-H\right) \geqslant 1.8 \mathrm{mag}\right)$ restrict our sample to have $z^{\prime} \leqslant 26.8 \mathrm{mag}$, which is roughly 0.5 mag brighter than the $z^{\prime}$-band limit (Dahlen et al. 2010). The uncertainties presented here reflect the Poisson variation in the object counts and do not include any contribution from cosmic variance.

GalFit typically overestimates the effective radii by $\sim 10 \%$ and underestimates the uncertainties by $\sim 10 \%$. Both the systematic bias and uncertainty are weakly brightness dependent, where fainter galaxies are more biased and uncertain. We remove the brightness-dependent bias and quadratically add the uncertainty to our effective radii measurements reported in Table 2.

Many of the these red galaxies are very small and, even with the space-based imaging, may still be unresolved. Therefore, it is critical to properly identify which galaxies are resolved and have reliable effective radii measurements. We begin by swapping the fully variable Sérsic model for the primary galaxy with a pure PSF model, which can only vary in position and brightness. However, as we have simultaneously fitted every object in our postage stamps, we anticipate that GalFit may incorrectly change the parameters of neighboring (unrelated) galaxies to compensate for the poor primary model, particularly in the case of a well-resolved primary galaxy. Therefore, when we use the PSF model for the primary galaxy, we hold the parameters of the neighboring sources fixed at the values found previously by GalFit. We now compare the goodness-of-fit statistics for these Sérsic and PSF models by considering the quantity:

$$
F=\frac{\chi_{\text {PSF }}^{2}-\chi_{\text {Sérsic }}^{2}}{\chi_{\text {Séarsic }}^{2}},
$$

and expect that sources with low values of $F$ are equally well characterized by a PSF model as by the more complex Sérsic profile. We calibrate this quantity by computing the $F$-values of the known ERS stars, which were used to derive the empirical PSF used above. In Figure 4, we show the $F$-values as a function of the GalFit-derived PSF magnitude, with the galaxies plotted as filled-blue points and the stars as red asterisks. The trend is as

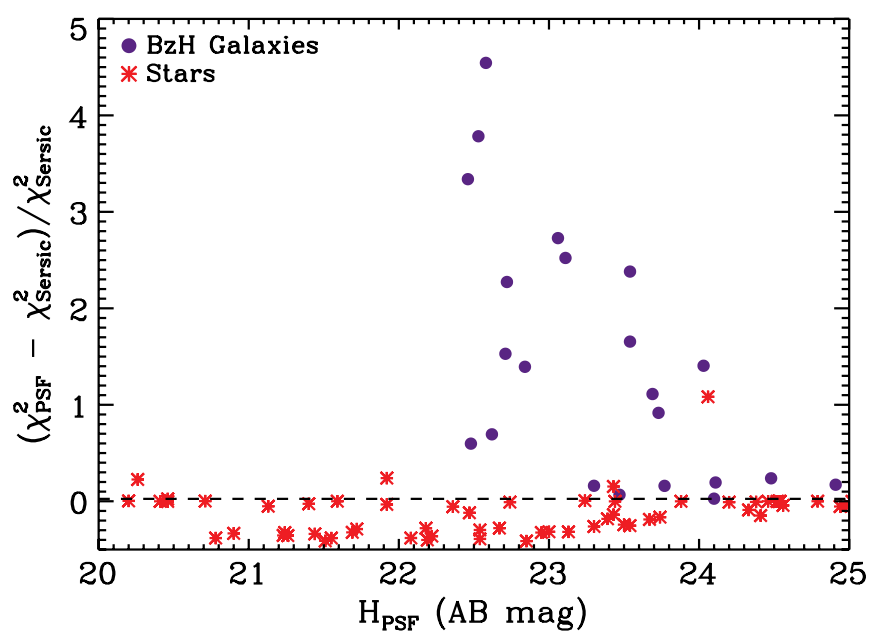

Figure 4. Comparison of PSF and Sérsic models. We show the fractional difference between the $\chi^{2}$ goodness-of-fit measures from GalFit for the PSF and Sérsic models. The $B z H$ galaxies are shown as filled-blue points, while the red asterisks represent 75 ERS point sources, presumed to be stars. For GalFit to optimally fit an unresolved source with a PSF, it will drive the effective radius and Sérsic index to unphysical regimes, de facto fitting a PSF. Therefore, the goodness of fit of a Sérsic model and a PSF should be roughly equal. However, if one were to fit a PSF to a resolved object, then there should be a noticeable increase in the goodness-of-fit statistic. We adopt a critical value of $F_{\text {crit }}=0.025$, see Section 4.1 for more details. Of the 75 stars used in this test, only 4 have $F>F_{\text {crit }}$ suggesting this is a reliable way to classify unresolved objects.

(A color version of this figure is available in the online journal.)

expected: nearly all point sources can be found at $-0.5 \lesssim F \lesssim 0$, while the $B z H$ galaxies are generally at $F \gtrsim 0$ which depends on brightness. Therefore, we define objects which can be equally characterized by a PSF as by a Sérsic fit as having $F \leqslant F_{\text {crit }}$, while objects with $F>F_{\text {crit }}$ are more extended than the known ERS stars. We adopt $F_{\text {crit }}=0.025$ and note that only $4 / 75$ stars have $F>F_{\text {crit }}$, which is consistent with Bond et al. (2009). We give the GalFit results in Table 2.

\subsection{Stellar Populations and Photometric Redshifts}

We fit the matched-aperture HST photometry with a library of stellar population synthesis models to simultaneously determine the stellar mass, population age, and redshift with our own software. Our model grid consists of a four-dimensional parameter space spanned by redshift $(z)$, stellar population age ${ }^{24}$ $(t), V$-band extinction $\left(A_{V}\right)$, and star formation timescale $(\tau)$ for an exponentially declining star formation history. We assume solar metallicity, a Salpeter initial mass function (IMF), and adopt the CB07 population synthesis models. The allowed photometric redshifts ranged from $0 \leqslant z<7$ with $\Delta z=0.01$, the ages presented by $\mathrm{CB} 07$, extinctions of $0 \mathrm{mag} \leqslant A_{V} \leqslant 2 \mathrm{mag}$ with $\Delta A_{V}=0.2 \mathrm{mag}$, and star formation timescales of $\tau \in$ $\left[10^{-3}, 1,2,3,5,15,30,10^{3}\right]$ Gyr. We include a $10 \%$ systematic uncertainty on the observed fluxes to account for uncertainties in the zero points and assumed templates. We compute the $1 \sigma$ uncertainties on the stellar population parameters (e.g., mass, age, etc.) by use of a simple Monte Carlo calculation. For each band for a given galaxy, we draw a normal random variable with mean and standard deviation equal to the flux and flux uncertainty (without the systematic term) and catalog the new population parameters. By repeating for $10^{4}$ iterations, we build

\footnotetext{
24 We impose the usual self-consistency constraint that the a galaxy be younger than the age of the universe.
} 
Table 2

$B z H$ GalFit Results

\begin{tabular}{|c|c|c|c|c|c|}
\hline ID & $\begin{array}{c}r_{e}{ }^{\mathrm{a}} \\
(\operatorname{arcsec})\end{array}$ & $n^{\mathrm{b}}$ & $\chi_{v}^{2}$ & $F^{\mathrm{c}}$ & Notes \\
\hline 408 & $0.04 \pm 0.01$ & $4.72 \pm 0.45$ & 0.487 & 0.160 & $\cdots$ \\
\hline 606 & $0.09 \pm 0.01$ & $6.92 \pm 0.92$ & 0.404 & 0.158 & $\cdots$ \\
\hline 1696 & $0.44 \pm 0.48$ & $1.21 \pm 0.10$ & 0.356 & 0.170 & $\ldots$ \\
\hline 2227 & $0.36 \pm 0.04$ & $2.93 \pm 0.09$ & 0.364 & 2.517 & $\ldots$ \\
\hline 2377 & $0.18 \pm 0.05$ & $4.17 \pm 1.01$ & 0.322 & 0.049 & $\cdots$ \\
\hline 2749 & $0.51 \pm 0.19$ & $0.57 \pm 0.05$ & 1.849 & 0.191 & $\cdots$ \\
\hline 2750 & $1.02 \pm 0.26$ & $5.65 \pm 0.58$ & 1.891 & 0.591 & $\cdots$ \\
\hline 2871 & $0.03 \pm 0.01$ & $8.00 \pm 2.76$ & 0.567 & 0.023 & Unresolved \\
\hline 3000 & $0.60 \pm 0.06$ & $8.00 \pm 0.14$ & 0.814 & 9.440 & Tidal tail—the fit is dubious \\
\hline 3152 & $0.14 \pm 0.01$ & $4.66 \pm 0.12$ & 0.515 & 3.323 & .. \\
\hline 3237 & $0.22 \pm 0.02$ & $7.54 \pm 0.30$ & 0.432 & 2.268 & $\ldots$ \\
\hline 3360 & $0.11 \pm 0.01$ & $7.58 \pm 0.46$ & 0.466 & 1.382 & $\ldots$ \\
\hline 3376 & $0.17 \pm 0.05$ & $2.21 \pm 0.44$ & 0.349 & 0.054 & $\cdots$ \\
\hline 3471 & $0.08 \pm 0.01$ & $4.40 \pm 0.16$ & 0.478 & 1.525 & $\cdots$ \\
\hline 3488 & $2.85 \pm 6.07$ & $0.73 \pm 0.13$ & 0.494 & 0.058 & $\ldots$ \\
\hline 3551 & $0.25 \pm 0.03$ & $8.00 \pm 0.22$ & 0.619 & 5.687 & Additional nuclear point source was included \\
\hline 3812 & $0.16 \pm 0.02$ & $3.12 \pm 0.07$ & 0.486 & 3.767 & $\cdots$ \\
\hline 4148 & $0.04 \pm 0.01$ & $5.73 \pm 0.32$ & 0.443 & 0.690 & $\ldots$ \\
\hline 4173 & $0.38 \pm 0.05$ & $0.98 \pm 0.02$ & 0.359 & 2.373 & $\cdots$ \\
\hline 4324 & $0.25 \pm 0.02$ & $3.27 \pm 0.08$ & 0.404 & 4.536 & $\cdots$ \\
\hline 4327 & $0.03 \pm 0.02$ & $2.96 \pm 3.62$ & 0.411 & 0.000 & Unresolved \\
\hline 4534 & $0.14 \pm 0.01$ & $2.21 \pm 0.07$ & 0.388 & 2.718 & $\ldots$ \\
\hline 4648 & $0.18 \pm 0.02$ & $2.08 \pm 0.10$ & 0.355 & 0.913 & $\ldots$ \\
\hline 4846 & $0.17 \pm 0.02$ & $1.30 \pm 0.13$ & 0.506 & 0.232 & $\ldots$ \\
\hline 4921 & $0.18 \pm 0.10$ & $8.00 \pm 4.50$ & 0.337 & 0.012 & Unresolved \\
\hline 5410 & $\cdots$ & $2.64 \pm 1.00$ & 0.364 & -0.009 & Likely three distinct clumps- the fit is dubious \\
\hline 5529 & $0.28 \pm 0.03$ & $2.63 \pm 0.12$ & 0.369 & 1.107 & $\cdots$ \\
\hline 5685 & $0.47 \pm 0.21$ & $0.36 \pm 0.02$ & 0.386 & 1.399 & $\cdots$ \\
\hline 5735 & $0.09 \pm 0.02$ & $8.00 \pm 4.15$ & 0.349 & 0.012 & $\cdots$ \\
\hline 6845 & $0.43 \pm 0.09$ & $1.33 \pm 0.04$ & 0.385 & 1.648 & $\cdots$ \\
\hline 7202 & $0.30 \pm 0.03$ & $2.29 \pm 0.04$ & 0.434 & 5.825 & $\cdots$ \\
\hline
\end{tabular}

Notes.

a The uncertainties on the effective radius include the systematic uncertainties determined in Section 4.1.

${ }^{\mathrm{b}}$ The Sérsic index in Equation (3).

${ }^{\mathrm{c}}$ The fractional difference between the goodness of fit for the PSF and Sérsic models (see Section 4.1 for more details).

up a distribution of each stellar population parameter and take the mean and standard deviation as the measured quantity and uncertainties, respectively.

We apply this approach to the complete sample of 30 $B z H$ galaxies. In Figure 5, we show the photometric redshift distribution and the comparison to a spectroscopic redshift, when available. As expected, the median redshift of the sample is $z_{\text {phot }}=1.6 \pm 0.6,{ }^{25}$ where the uncertainty reflects the standard deviation of the distribution. To estimate our typical redshift uncertainty, we compare to the published spectroscopic redshifts in the lower panel of Figure 5. Based on the root-mean-squared scatter of the seven objects with known spectroscopic redshifts, we estimate our uncertainties in $(1+z)$ are $\sim 3.3 \%$. We do recover many of the known galaxy cluster members at $z \sim 1.6$ (Kurk et al. 2009). We present our resulting photometric redshifts and stellar population parameters in Table 3 .

\section{5. $B z H$ GALAXY SIZE EVOLUTION}

Based on the photometric redshift estimates and the spectroscopic data (where available), the $B z H$ selection reliably identifies galaxies in the interval $z_{\text {phot }} \sim 1.6$. However, to study

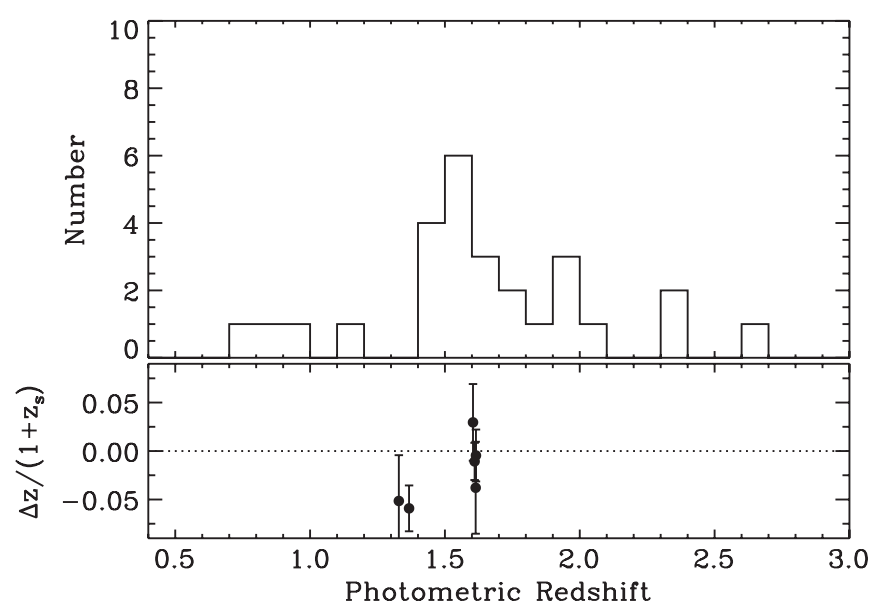

Figure 5. Photometric redshift distribution. In the upper panel, we show the photometric redshift distribution derived by the procedure described in Section 4.2. As expected, our sample of $B z H$ galaxies is generally located at $z_{\text {phot }}=1.6$. In the lower panel, we show the fractional difference between our redshift estimates and spectroscopic redshifts (where available) as a function of their photometric redshift. Based on the rms on the fractional differences, we estimate that $(1+z)$ is accurate to $\sim 3.3 \%$.

\footnotetext{
25 This could be potentially biased given the presence of a known overdensity
} at $z \sim 1.6$ (Kurk et al. 2009). 
Table 3

Photometric Redshifts and Stellar Population Parameters

\begin{tabular}{|c|c|c|c|c|c|}
\hline ID & $z_{\text {phot }}$ & $z_{\text {spec }}$ & $\begin{array}{c}\log \text { Age }^{a} \\
(\log y r)\end{array}$ & $\begin{array}{c}\log \text { Mass }^{\mathrm{b}} \\
\left(\log M_{\odot}\right)\end{array}$ & $\chi_{v}^{2}$ \\
\hline 408 & $1.58 \pm 0.05$ & $\ldots$ & $8.7 \pm 0.2$ & $10.6 \pm 0.1$ & 0.9 \\
\hline 606 & $1.89 \pm 0.19$ & $\ldots$ & $8.8 \pm 0.3$ & $10.6 \pm 0.2$ & 1.9 \\
\hline 1696 & $3.78 \pm 0.83$ & $\cdots$ & $8.7 \pm 0.2$ & $11.4 \pm 0.7$ & 3.3 \\
\hline 2227 & $0.79 \pm 0.44$ & $\ldots$ & $9.1 \pm 0.0$ & $9.9 \pm 0.6$ & 4.8 \\
\hline 2377 & $2.35 \pm 0.20$ & $\cdots$ & $8.7 \pm 0.3$ & $10.5 \pm 0.7$ & 3.0 \\
\hline 2749 & $1.96 \pm 0.20$ & $\cdots$ & $8.4 \pm 0.5$ & $10.9 \pm 0.2$ & 4.2 \\
\hline 2750 & $1.90 \pm 0.10$ & $\cdots$ & $8.2 \pm 0.3$ & $11.1 \pm 0.2$ & 6.8 \\
\hline 2871 & $1.52 \pm 0.05$ & $\cdots$ & $8.7 \pm 0.2$ & $10.4 \pm 0.1$ & 2.2 \\
\hline 3000 & $1.50 \pm 0.05$ & $\cdots$ & $8.9 \pm 0.1$ & $11.5 \pm 0.1$ & 0.6 \\
\hline 3152 & $1.51 \pm 0.06$ & 1.367 & $9.0 \pm 0.1$ & $11.2 \pm 0.1$ & 1.4 \\
\hline 3237 & $1.63 \pm 0.07$ & 1.615 & $8.8 \pm 0.3$ & $11.1 \pm 0.1$ & 1.9 \\
\hline 3360 & $1.73 \pm 0.20$ & $\cdots$ & $8.9 \pm 0.1$ & $11.1 \pm 0.1$ & 0.9 \\
\hline 3376 & $1.48 \pm 0.09$ & $\cdots$ & $8.5 \pm 0.3$ & $9.8 \pm 0.2$ & 0.9 \\
\hline 3471 & $1.64 \pm 0.05$ & 1.610 & $8.9 \pm 0.1$ & $10.9 \pm 0.1$ & 1.0 \\
\hline 3488 & $4.73 \pm 0.29$ & $\cdots$ & $8.7 \pm 0.1$ & $11.4 \pm 0.4$ & 2.5 \\
\hline 3551 & $1.58 \pm 0.06$ & $\cdots$ & $9.0 \pm 0.1$ & $11.3 \pm 0.1$ & 1.7 \\
\hline 3812 & $1.71 \pm 0.12$ & 1.614 & $9.0 \pm 0.1$ & $11.0 \pm 0.1$ & 1.7 \\
\hline 4148 & $2.36 \pm 0.04$ & $\cdots$ & $8.6 \pm 0.1$ & $11.0 \pm 0.1$ & 2.9 \\
\hline 4173 & $0.98 \pm 0.42$ & $\cdots$ & $9.0 \pm 0.3$ & $10.1 \pm 0.7$ & 1.2 \\
\hline 4324 & $1.54 \pm 0.08$ & $\cdots$ & $9.0 \pm 0.1$ & $10.9 \pm 0.1$ & 2.7 \\
\hline 4327 & $3.13 \pm 0.76$ & $\cdots$ & $8.6 \pm 0.3$ & $10.9 \pm 0.8$ & 1.8 \\
\hline 4534 & $1.53 \pm 0.10$ & 1.604 & $9.0 \pm 0.1$ & $10.8 \pm 0.1$ & 1.8 \\
\hline 4648 & $0.86 \pm 0.62$ & $\cdots$ & $8.8 \pm 0.4$ & $9.7 \pm 0.8$ & 2.5 \\
\hline 4846 & $1.63 \pm 0.10$ & $\cdots$ & $8.8 \pm 0.2$ & $10.4 \pm 0.2$ & 3.5 \\
\hline 4921 & $3.57 \pm 0.88$ & $\cdots$ & $8.8 \pm 0.2$ & $11.4 \pm 1.0$ & 2.6 \\
\hline 5410 & $1.99 \pm 0.18$ & $\cdots$ & $8.5 \pm 0.3$ & $10.4 \pm 0.3$ & 4.7 \\
\hline 5529 & $1.17 \pm 0.41$ & $\cdots$ & $9.0 \pm 0.1$ & $10.4 \pm 0.2$ & 1.0 \\
\hline 5685 & $1.48 \pm 0.07$ & $\cdots$ & $9.0 \pm 0.1$ & $10.3 \pm 0.2$ & 0.9 \\
\hline 5735 & $2.66 \pm 0.12$ & $\cdots$ & $8.4 \pm 0.1$ & $9.9 \pm 0.2$ & 1.0 \\
\hline 6845 & $2.03 \pm 0.53$ & $\cdots$ & $8.9 \pm 0.2$ & $10.8 \pm 0.2$ & 1.0 \\
\hline 7202 & $1.45 \pm 0.11$ & 1.329 & $8.4 \pm 0.1$ & $11.0 \pm 0.2$ & 1.2 \\
\hline
\end{tabular}

Notes.

a Population age assuming an exponential star formation history.

b Stellar mass.

the evolution of their sizes with redshift, we must compare to other similarly selected samples. The high-redshift $(z \gtrsim 1.5)$ samples are generally derived from similar color criteria presented here and have effective radii measured in the $H$ band, for a rest-frame wavelength of $\lambda_{\text {rest }} \sim 6500 \AA$. To ensure fair comparisons with lower redshift samples, we require sizes measured at similar rest-frame wavelengths. For the low-redshift data, we use the sample of 8666 early-type galaxies at $z \sim 0.2$ with effective radii measured in the $i^{\prime}$ band (Bernardi et al. 2003) selected from the Sloan Digital Sky Survey, Early Data Release (SDSS-EDR; York et al. 2000). The stellar masses for the 7 th Data Release ${ }^{26}$ galaxies were determined following Salim et al. (2007). By cross matching these samples, we obtain 8595 galaxies suitable for our low-redshift comparison. We select three mass ranges which are volume limited based on the upper and lower flux limits imposed by detector saturation in SDSS and the Bernardi et al. (2003) brightness criterion, respectively. In Figure 6, we show these volume-limited selections (black boxes) and the flux limits (dashed lines). If these limits are not strictly imposed, then an artificial redshift dependence on the effective radii will be introduced as the radii are tightly correlated with the stellar masses, which roughly scale with luminosity. For example, without these volume limits, the effective radii of the SDSS galaxies will seem to increase with redshift since the

\footnotetext{
26 Obtained from http://www.mpa-garching.mpg.de/SDSS/DR7/.
}

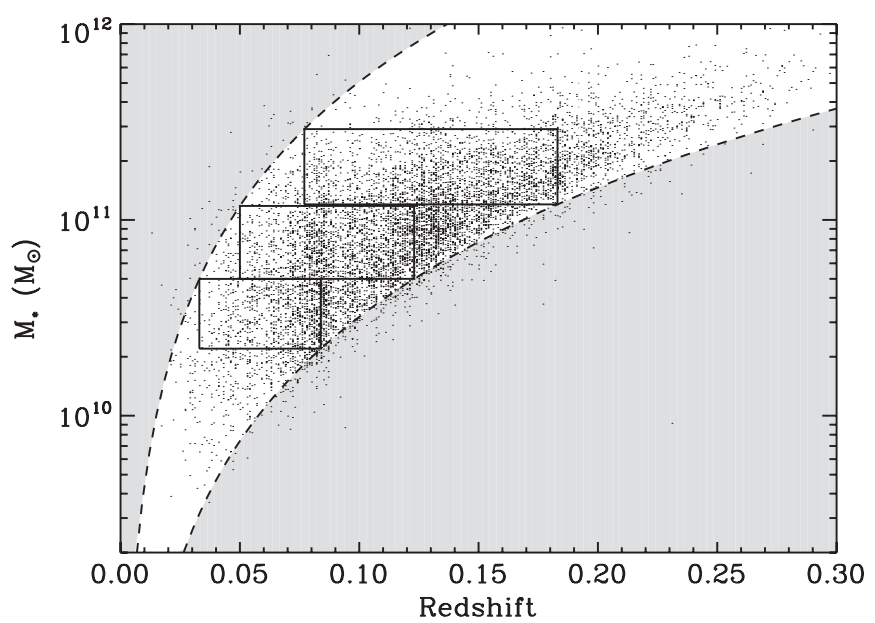

Figure 6. Selection of SDSS galaxy sample. We show the stellar mass as a function of spectroscopic redshift for the early-type galaxy sample of Bernardi et al. (2003) drawn from the SDSS-EDR. The stellar mass estimates were derived from stellar population fits to the SDSS photometry (Salim et al. 2007) and are similar in nature to those described in Section 4.2. The dashed lines indicate the bright $\left(i^{\prime} \leqslant 14 \mathrm{mag}\right)$ and faint $\left(i^{\prime} \geqslant 17 \mathrm{mag}\right)$ completeness limits set by detector saturation and the Bernardi et al. (2003) selection, respectively. The boxes show our volume-limited selections for proper low-redshift comparisons. If such limits are not imposed, then an artificial trend in effective radius with redshift will arise from a Malmquist-type bias.

survey is not sensitive to the lower mass (smaller) galaxies at the higher redshifts. Eliminating this critical Malmquist-like bias in the low-redshift sample ensures a fair comparison between the high- and low-redshift data.

To investigate the passively evolving galaxy size evolution at a fixed stellar mass, we show in Figure 7 the effective radii as a function of redshift for this work (filled circles) and a host of published measurements as described in the caption. We convert all mass estimates from the literature to the Salpeter IMF based on corrections discussed in the Appendix. For clarity, we do not show three objects whose measured effective radii are $r_{e} \leqslant 0.5$ pixel as marked by the gray shaded region. While these objects are formally consistent with being extended, such small sizes are dubious and highly degenerate with the Sérsic index and local sky estimation. Furthermore, there are 11 objects from our sample which are either outside our mass range $\left(10.3 \leqslant \log \left(M_{*} / M_{\odot}\right) \leqslant 11.5\right)$ or have photometric redshifts at $z_{\text {phot }} \geqslant 2.6$, as the spectral energy distribution fits are highly suspect. We overplot the power-law models of the form $R_{e} \propto(1+z)^{-\alpha}$ (thick black line) where $\alpha$ is determined for each mass range separately and present the goodness-offit statistic for the two models in the lower-left corner of each panel. We treat the canon of SDSS as a single low-redshift constraint, using their mean and error in the mean for the fits. For comparison, we show the mass-independent model (dashed blue line) in each panel, which was determined by fitting the data in all three panels simultaneously. We note that fits of the form $R_{e} \propto H(z)^{-\beta}$ (where $H(z)$ is the Hubble parameter) give a similar qualitative result: the amount by which galaxies are smaller in the past depends on their stellar mass. Newman et al. (2010) identified a similar result for 12 galaxies with dynamical masses determined from measured velocity dispersions.

Our preliminary estimate of a mass-dependent size evolution model is qualitatively similar to the predictions from the semianalytic models of Hopkins et al. (2009), where the powerlaw index varies with mass as $\alpha\left(M_{*}\right) \approx 0.23 \log \left(M_{*} / 10^{9} M_{\odot}\right)$. However, their model underpredicts our measured power-law 


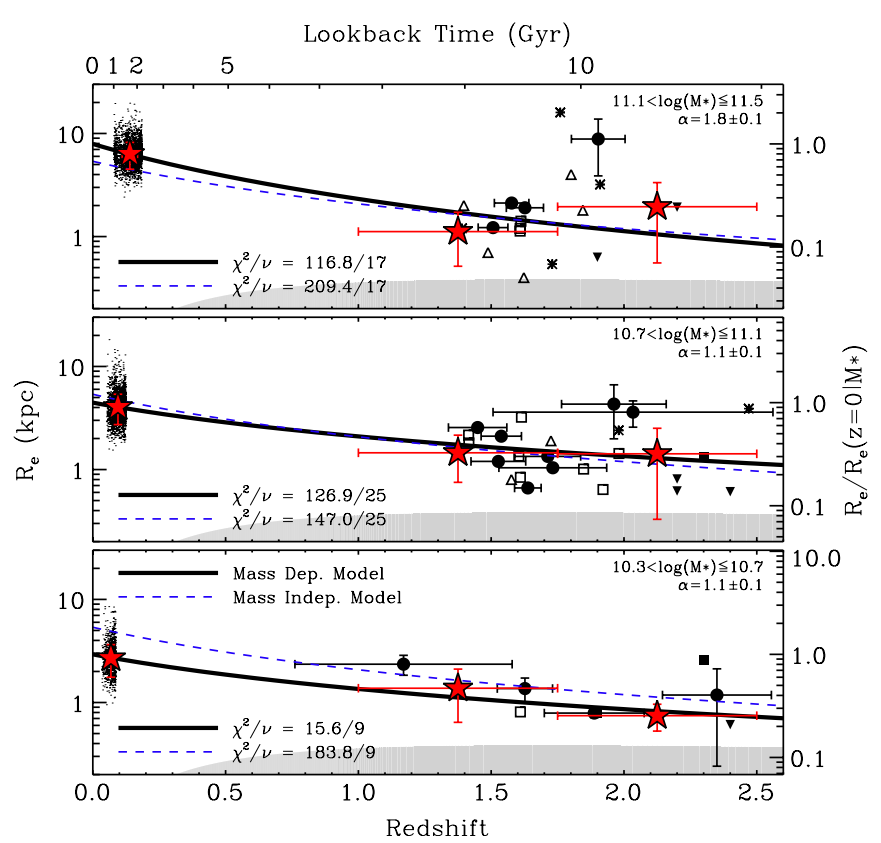

Figure 7. $B z H$ galaxy size evolution. We show the effective radius vs. redshift for the various stellar mass slices described in Figure 6 and several earlytype galaxy samples: SDSS sample (Bernardi et al. 2003; Salim et al. 2007; small dots), Daddi et al. (2005; asterisks), Longhetti et al. (2007; diamonds), Toft et al. (2007; downward triangles), Zirm et al. (2007; closed squares), Cimatti et al. (2008; open squares), Damjanov et al. (2009; upward triangles), Carrasco et al. (2010; open circles), and this work as large-filled circles. All stellar masses assume a Salpeter IMF with solar metallicity (see Section 5 and the Appendix for the conversion factors). We show the power-law fit $R_{e} \propto(1+z)^{-\alpha}$, where $\alpha$ is determined separately for each mass bin (thick black line) and for all bins together (dashed blue line), and report the reduced $\chi^{2}$ for each model in the lower left of each panel. The gray shaded region in each panel indicates the position of unresolved objects with $r_{e} \lesssim 0.5$ pixel; we omit three objects which are marginally resolved (based on their $F$-ratios), but are within this region. The red stars indicate the average and rms values in three redshift intervals, which are only used to illustrate the trend. Based on the reduced $\chi^{2}$ values, we conclude the mass-dependent model (full black line) better reproduces the relationship between the effective radius and redshift than the mass-independent (dashed blue line), while preserving the local $R_{e}-M_{*}$ trend (e.g., Shen et al. 2003). Since the majority of high-redshift objects have very similar effective radii, stellar mass, and redshift distributions, much of the variance in $\alpha$ is set by the SDSS sample. However, larger high-redshift samples (comparable in size to those at low redshift) are needed to assess their relative importance in setting the mass dependence on $\alpha$.

(A color version of this figure is available in the online journal.)

indices in our three mass regimes, but does give the same qualitative steepening of $\alpha$ with mass. Following their approach, we derive tentative relationships for the power-law indices:

$$
\alpha\left(M_{*}\right) \approx(-0.6 \pm 0.7)+(0.9 \pm 0.4) \times \log \left(\frac{M_{*}}{10^{9} M_{\odot}}\right)
$$

and caution that with only three independent mass bins, this scaling should be considered preliminary at best. However, this result gives indices consistent with the reported value of Buitrago et al. (2008), who find that $\alpha\left(M_{*}>10^{11} M_{\odot}\right) \approx$ 1.5. In Section 7 , we discuss some potential shortcomings of the current data and propose experiments for future data sets.

\section{DISCUSSION}

It has become relatively well established that early-type galaxies are indeed smaller at high redshift than their local counterparts for a given stellar or dynamical mass (e.g., Trujillo et al. 2009; Newman et al. 2010). However, the causes for this result are far less clear, or agreed upon. The likely mechanisms can be broadly characterized as astrophysical effects (early-time major mergers, late-time minor mergers, adiabatic expansion, or stellar mass-to-light gradients), or observational biases (underestimating the effective radii, overestimating the stellar masses, or incorrectly assuming that the high- and lowredshift populations are directly comparable). Using a semianalytical model, Hopkins et al. (2010) find that the factor of $\sim 5$ increase in the effective radii over the last $\sim 10 \mathrm{Gyr}$ for galaxies with $M_{*} \geqslant 10^{11} M_{\odot}$ can be explained by a combination of these effects, with the late-time minor merging playing the largest role. However, we find that lower mass systems exhibit notably weaker redshift evolution, suggesting a different mixture of the main processes may be at work. For example, the adiabatic expansion mode may become more critical given the shallower gravitational potentials in the low-mass systems.

As noted above, the tentative stellar mass-dependent size evolution presented in this work is similar to the findings of Newman et al. (2010) for dynamical masses estimated from velocity dispersions, and has an interesting consequence for the $R_{e}-M_{*}$ relation. Shen et al. (2003) find that SDSS elliptical galaxies follow the scaling relation $R_{e}=R_{e, 11}\left(M_{*} / 10^{11} M_{\odot}\right)^{\gamma}$, where $R_{e, 11}=4.16 \mathrm{kpc}$ and $\gamma=0.56$. However, the massdependent size evolution found by us and by Newman et al. (2010) imply either a fundamental change in the $R_{e}-M$ scaling relationship at high redshift (such as a flattening of the effective radius for decreasing stellar mass) or a redshift-dependent value of $\gamma$, in addition to the usual lower value of $R_{e, 11}$. Unfortunately, at this stage the high-redshift data cannot distinguish these two scenarios or shed light on the cause for this change. In fact, given the large scatter in $R_{e}-M_{*}$ at high redshift $(z \sim 1.5)$, most of the size evolution is driven by the Bernardi et al. (2003) sample at $z \sim 0.2$.

The favored red galaxy formation paradigm suggests that passively evolving galaxies have come from a population of more active systems in their recent past. These systems originate from mergers of gas-rich disks that trigger intense starbursts which are later quenched by active galactic nuclei (AGNs), finally resulting in dead spheroidal systems spent of their gas and no longer forming stars (e.g., Faber et al. 2007, and references therein). The Lyman break galaxies (LBGs) observed at $z \gtrsim 3$ are a possible progenitor system (e.g., Overzier et al. 2010). While their stellar masses are far less well constrained due to the lack of rest-frame IR data, the majority of LBGs have $M_{*} \sim 10^{10} M_{\odot}$ between $3 \lesssim z \lesssim 6$ (e.g., Papovich et al. 2001; Yan et al. 2006). Therefore, the typical LBG at $3 \lesssim z \lesssim 6$ belongs to the lower panel of Figure 7 and has effective radii of $R_{e} \sim 1 \mathrm{kpc}$ (e.g., Ferguson et al. 2004; Bouwens et al. 2006; Hathi et al. 2008; Oesch et al. 2010), after transforming to an equivalent rest-frame wavelength (Barden et al. 2005). The LBGs are then consistent with our size evolution model, possibly suggesting that similar physical mechanisms driving the passive galaxy evolution may be at work for the LBGs as well. Given their increased star formation rates, larger gas content, and lower total mass, the mass-loss modes, whether driven by AGNs (e.g., Fan et al. 2008) or stellar winds (e.g., Damjanov et al. 2009), are potentially more important. However, we recognize that these samples (LBGs and passive galaxies) are quite different and that the next generation of space-based infrared instruments (Near-Infrared Camera and Mid-Infrared 
Table 4

Stellar Mass Transformations ${ }^{\mathrm{a}}$

\begin{tabular}{|c|c|c|c|c|c|c|c|c|c|c|c|c|}
\hline & & \multicolumn{11}{|c|}{$\log M_{*, i}$} \\
\hline & & $35 \mathrm{c}$ & $35 \mathrm{~s}$ & $36 \mathrm{c}$ & $36 \mathrm{~s}$ & $37 \mathrm{c}$ & $37 \mathrm{~s}$ & $75 \mathrm{c}$ & $75 \mathrm{~s}$ & $76 \mathrm{c}$ & $76 \mathrm{~s}$ & $77 \mathrm{c}$ \\
\hline \multirow{11}{*}{$\log M_{*, j}$} & $35 \mathrm{~s}$ & -0.25 & $\ldots$ & $\ldots$ & $\ldots$ & $\ldots$ & $\ldots$ & $\ldots$ & $\ldots$ & $\ldots$ & $\ldots$ & $\cdots$ \\
\hline & $36 \mathrm{c}$ & +0.06 & +0.31 & $\ldots$ & $\ldots$ & $\ldots$ & $\ldots$ & $\ldots$ & $\ldots$ & $\ldots$ & $\ldots$ & $\ldots$ \\
\hline & $36 \mathrm{~s}$ & -0.19 & +0.06 & -0.24 & $\ldots$ & $\cdots$ & $\ldots$ & $\cdots$ & $\cdots$ & $\cdots$ & $\ldots$ & $\cdots$ \\
\hline & $37 \mathrm{c}$ & +0.11 & +0.36 & +0.05 & +0.29 & $\ldots$ & $\ldots$ & $\ldots$ & $\ldots$ & $\ldots$ & $\ldots$ & $\ldots$ \\
\hline & $37 \mathrm{~s}$ & -0.14 & +0.11 & -0.20 & +0.04 & -0.24 & $\ldots$ & $\ldots$ & $\ldots$ & $\ldots$ & $\ldots$ & $\ldots$ \\
\hline & $75 \mathrm{c}$ & +0.07 & +0.32 & +0.01 & +0.25 & -0.04 & +0.20 & $\cdots$ & $\ldots$ & $\ldots$ & $\ldots$ & $\cdots$ \\
\hline & $75 \mathrm{~s}$ & -0.17 & +0.08 & -0.24 & +0.01 & -0.29 & -0.04 & -0.25 & $\ldots$ & $\ldots$ & $\ldots$ & $\ldots$ \\
\hline & $76 \mathrm{c}$ & +0.06 & +0.31 & -0.00 & +0.24 & -0.06 & +0.19 & -0.01 & +0.24 & $\ldots$ & $\ldots$ & $\ldots$ \\
\hline & $76 \mathrm{~s}$ & -0.18 & +0.07 & -0.25 & -0.00 & -0.30 & -0.05 & -0.25 & -0.01 & -0.24 & $\ldots$ & $\ldots$ \\
\hline & $77 \mathrm{c}$ & +0.09 & +0.34 & +0.02 & +0.27 & -0.01 & +0.24 & +0.02 & +0.27 & +0.03 & +0.27 & $\ldots$ \\
\hline & $77 \mathrm{~s}$ & -0.16 & +0.09 & -0.22 & +0.03 & -0.25 & -0.01 & -0.22 & +0.02 & -0.22 & +0.03 & -0.24 \\
\hline
\end{tabular}

Note.

${ }^{\text {a }}$ We report the quantity $\left\langle\log \frac{M_{*, i}}{M_{*, j}}\right\rangle$.

Instrument on James Webb Space Telescope, JWST) will provide a much clearer picture for the high-redshift $(z \gtrsim 3)$ size comparisons.

\section{SUMMARY}

We identified 30 passively evolving galaxies to $H \leqslant 25$ mag from a set of color criteria similar to the $\mathrm{p} B z K$ selection. We measure rest-frame optical $\left(\lambda_{\text {rest }} \sim 6500 \AA\right)$ effective radii as two-dimensional fits to the $H$-band image. By comparing with several other comparable samples at similar redshifts, we find that the size evolution depends on the stellar mass. We give tentative scalings between the power-law index of the $R_{e}-z$ relation and stellar mass. While we have made every attempt at matching the selection biases between the high- and lowredshift samples, subtle differences may remain (such as the requirements on spectroscopic redshift and visual classification on the low-redshift data not matched at high redshift). Given the various requirements on placing SDSS fibers and the seeinglimited imaging, it is easy to believe that there may be systematic differences in the way the samples are created. Using future surveys with ultraviolet imaging with HST (such as planned observations with CANDELS and of the Hubble Ultra-Deep Field), we can extend the redshift range to $0.3 \lesssim z \lesssim 1.3$ while consistently selecting galaxies of identical rest-frame spectral properties and removing any potential differences between the high- and low-redshift samples.

Special thanks are due to D. Wittman, P. Gee, C. Peng, J. Bosch, S. Schmidt, and P. Thorman. We are grateful to the men and women who worked tirelessly for many years to make Wide-Field Camera 3 the instrument it is today, and to the STScI Director M. Mountain for the discretionary time to make this program possible. We thank the anonymous referee for their excellent comments and suggestions. R.E.R. acknowledges support from NASA through grant number 11772 and for $H S T$ program 11359 was provided by NASA through grants GO11359.0.A from the Space Telescope Science Institute, which is operated by the Association of Universities for Research in Astronomy, Inc., under NASA contract NAS 5-26555. R.A.W. acknowledges support from NASA JWST Interdisciplinary Scientist grant NAG5-12469 from GSFC. Finally, we are deeply indebted to the brave astronauts of STS-125 for upgrading and extending $H S T$ into the future.

Facilities: HST (WFC3, ACS)

\section{APPENDIX \\ STELLAR MASS CONVERSIONS}

In most cases when authors determine stellar masses from population synthesis techniques, they adopt a single IMF and metallicity with the understanding that the stellar masses are dependent on these choices. Since there is no clear indication of which IMF may be appropriate, and modeling an arbitrary IMF and metallicity with broadband observations is prone to large degeneracies (Conroy et al. 2009), we are left to convert all stellar masses taken from the literature to a common set of assumptions. To derive these conversions, we refit our 30 objects with a different IMF and metallicity and compute

$$
\left\langle\log \frac{M_{*, i}}{M_{*, j}}\right\rangle=C_{i j},
$$

where $M_{*, i}$ and $M_{*, j}$ are the resulting stellar masses for the different input models $i$ and $j$, respectively. We extend this analysis to include the Bruzual \& Charlot (2003, BC03) models, which did not include flux contributions for thermally pulsating asymptotic giant branch stars. In Table 4 , we tabulate the $\mathbf{C}$ matrix to convert the mass estimates obtained from the literature (as discussed in Section 5), where a model is encoded by three characters: the first indicates the vintage $(3=\mathrm{BC} 03$ and $7=\mathrm{CB} 07)$, the second indicates the metallicity $\left(5=0.4 Z_{\odot}\right.$, $6=1.0 Z_{\odot}$, and $\left.7=2.5 Z_{\odot}\right)$, and the third indicates the IMF ( $\mathrm{S}=$ Salpeter or $\mathrm{C}=$ Chabrier $)$.

\section{REFERENCES}

Barden, M., Rix, H.-W., Somerville, R. S., et al. 2005, ApJ, 635, 959 Bell, E. F., Naab, T., McIntosh, D. H., et al. 2006, ApJ, 640, 241 Bell, E. F., Wolf, C., Meisenheimer, K., et al. 2004, ApJ, 608, 752 Bernardi, M., Sheth, R. K., Annis, J., et al. 2003, AJ, 125, 1817 Bertin, E., \& Arnouts, S. 1996, A\&AS, 117, 393

Bond, N. A., Gawiser, E., Gronwall, C., et al. 2009, ApJ, 705, 639 Bouwens, R. J., Illingworth, G. D., Blakeslee, J. P., \& Franx, M. 2006, ApJ, 653,53

Bremer, M. N., Valtchanov, I., Willis, J., et al. 2006, MNRAS, 371, 1427

Brown, M. J. I., Dey, A., Jannuzi, B. T., et al. 2003, ApJ, 597, 225

Brown, M. J. I., Dey, A., Jannuzi, B. T., et al. 2007, ApJ, 654, 858

Bruzual, G. 2007, in ASP Conf. Ser. 374, From Stars to Galaxies: Building the Pieces to Build Up the Universe, ed. A. Vallenari, R. Tantalo, L. Portinari, \& A. Moretti (San Francisco, CA: ASP), 303

Bruzual, G., \& Charlot, S. 2003, MNRAS, 344, 1000

Buitrago, F., Trujillo, I., Conselice, C. J., et al. 2008, ApJ, 687, L61 
Calzetti, D., Kinney, A. L., \& Storchi-Bergmann, T. 1994, ApJ, 429, 582

Carrasco, E. R., Conselice, C. J., \& Trujillo, I. 2010, MNRAS, 405, 2253

Cimatti, A., Cassata, P., Pozzetti, L., et al. 2008, A\&A, 482, 21

Cimatti, A., Daddi, E., \& Renzini, A. 2006, A\&A, 453, L29

Cimatti, A., Daddi, E., Renzini, A., et al. 2004, Nature, 430, 184

Coleman, G. D., Wu, C.-C., \& Weedman, D. W. 1980, ApJS, 43, 393

Conroy, C., Gunn, J. E., \& White, M. 2009, ApJ, 699, 486

Cox, T. J., Di Matteo, T., Hernquist, L., et al. 2006, ApJ, 650, 791

Daddi, E., Cimatti, A., Renzini, A., et al. 2004, ApJ, 617, 746

Daddi, E., Renzini, A., Pirzkal, N., et al. 2005, ApJ, 626, 680

Dahlen, T., Mobasher, B., Dickinson, M., et al. 2010, ApJ, 724, 425

Damjanov, I., McCarthy, P. J., Abraham, R. G., et al. 2009, ApJ, 695, 101

Demarco, R., Wilson, G., Muzzin, A., et al. 2010, ApJ, 711, 1185

Dickinson, M., Stern, D., Giavalisco, M., et al. 2004, ApJ, 600, L99

Djorgovski, S., \& Davis, M. 1987, ApJ, 313, 59

Dressler, A., Lynden-Bell, D., Burstein, D., et al. 1987, ApJ, 313, 42

Eggen, O. J., Lynden-Bell, D., \& Sandage, A. R. 1962, ApJ, 136, 748

Faber, S. M., Willmer, C. N. A., Wolf, C., et al. 2007, ApJ, 665, 265

Fan, L., Lapi, A., de Zotti, G., \& Danese, L. 2008, ApJ, 689, L101

Ferguson, H. C., Dickinson, M., Giavalisco, M., et al. 2004, ApJ, 600, L107

Ferreras, I., Pasquali, A., Khochfar, S., et al. 2011, arXiv:1109.6323

Giavalisco, M., Ferguson, H. C., Koekemoer, A. M., et al. 2004, ApJ, 600, L93

Glazebrook, K., Abraham, R. G., McCarthy, P. J., et al. 2004, Nature, 430, 181

Hathi, N. P., Malhotra, S., \& Rhoads, J. E. 2008, ApJ, 673, 686

Hopkins, P. F., Bundy, K., Hernquist, L., Wuyts, S., \& Cox, T. J. 2010, MNRAS, 401, 1099

Hopkins, P. F., Hernquist, L., Cox, T. J., Keres, D., \& Wuyts, S. 2009, ApJ, 691, 1424

Kalirai, J. S., MacKenty, J., Bohlin, R., et al. 2009b, in Instrument Science Report WFC3, WFC3 SMOV Proposal 11451: The Photometric Performance and Calibration of WFC3/IR, 2009-30

Kalirai, J. S., MacKenty, J., Rajan, A., et al. 2009a, in Instrument Science Report WFC3, WFC3 SMOV Proposal 11450: The Photometric Performance and Calibration of WFC3/UVIS, 2009-31
Koekemoer, A. M., Fruchter, A. S., Hook, R. N., \& Hack, W. 2002, in The 2002 HST Calibration Workshop: Hubble after the Installation of the ACS and the NICMOS Cooling System, ed. S. Arribas, A. Koekemoer, \& B. Whitmore (Baltimore, MD: STScI), 337

Kormendy, J. 1977, ApJ, 218, 333

Kriek, M., van der Well, A., van Dokkum, P. G., Franx, M., \& Illingworth, G. 2008, ApJ, 682, 896

Kurk, J., Cimatti, A., Zamorani, G., et al. 2009, A\&AS, 504, 331

Lane, K. P., Almaini, O., Foucaud, S., et al. 2007, MNRAS, 379, L25

Longhetti, M., Saracco, P., Severgnini, P., et al. 2007, MNRAS, 374, 614

Luo, B., Bauer, F. E., Brandt, W. N., et al. 2008, ApJS, 179, 19

McCarthy, P. J., Le Borgne, D., Crampton, D., et al. 2004, ApJ, 614, L9

Naab, T., Johansson, P. H., \& Ostriker, J. P. 2009, ApJ, 699, L178

Naab, T., Khochfar, S., \& Burkert, A. 2006, ApJ, 636, L81

Newman, A. B., Ellis, R. S., Treu, T., \& Bundy, K. 2010, ApJ, 717, 103

Oesch, P. A., Bouwens, R. J., Illingworth, G. D., et al. 2010, ApJ, 709, L16

Oke, J. B., \& Gunn, J. E. 1983, ApJ, 266, 713

Overzier, R. A., Heckman, T. M., Schiminovich, D., et al. 2010, ApJ, 701, 979

Papovich, C., Dickinson, M., \& Ferguson, H. C. 2001, ApJ, 559, 620

Peng, C., Ho, L. C., Impey, C. D., \& Rix, H.-W. 2002, AJ, 124, 266

Pickles, A. J. 1998, PASP, 110, 863

Rudnick, G., Rix, H.-W., Franx, M., et al. 2003, ApJ, 599, 847

Salim, S., Rich, R. M., Charlot, S., et al. 2007, ApJS, 173, 267

Saracco, P., Longhetti, M., \& Gargiulo, A. 2010, MNRAS, 408, L21

Shen, S., Mo, H. J., White, S. D. M., et al. 2003, MNRAS, 343, 978

Spergel, D. N., Verde, L., Peiris, H. V., et al. 2003, ApJS, 148, 175

Stutz, A. M., Papovich, C., \& Eisenstein, D. J. 2008, ApJ, 677, 828

Taylor, E. N., Franx, M., Glazebrook, K., et al. 2010, ApJ, 720, 723

Toft, S., van Dokkum, P., Franx, M., et al. 2007, ApJ, 671, 285

Trujillo, I., Cenarro, A. J., de Lorenzo-Cáceres, A., et al. 2009, ApJ, 692, L118

Trujillo, I., Feulner, G., Goranova, Y., et al. 2006, MNRAS, 373, L36

van Dokkum, P. G., \& Brammer, G. 2010, ApJ, 718, L73

van Dokkum, P. G., Franx, M., Kriek, M., et al. 2008, ApJ, 677, L5

Windhorst, R. A., Cohen, S. H., Hathi, N. P., et al. 2011, ApJS, 193, 27

Yan, H., Dickinson, M., Giavalisco, M., et al. 2006, ApJ, 651, 24

York, D. G., Adelman, J., Anderson, J. E., Jr., et al. 2000, AJ, 120, 1579

Zirm, A. W., van der Wel, A., Franx, M., et al. 2007, ApJ, 656, 66 